\title{
Super-nonradiative states in fractal quantum systems
}

\author{
Valeriy Abramov* \\ Galkin Institute of Physics and Technology, 83114 Donetsk, Ukraine
}

\begin{abstract}
Theoretically the relationships of the main parameters of active nanoobjects with the Higgs boson and the Higgs field in a fractal quantum systems, super-nonradiative states of physical fields are investigated. The estimations of numbers, mass and rest energy of the neutrino (solar, electronic), the rest energy of Majorana neutrino, dark matter are obtained.
\end{abstract}

Based on the hypothesis of the hierarchical structure of the Cosmos [1, 2], we can use experimental results in cosmology: for studying of anisotropic models of fractal cosmological objects; superradiance [1] of gravitational waves, relic photons from binary black holes, neutron stars; the nature of dark matter particles and dark energy [3], the chiral fractal structures of the Universe. In [2] a specific fundamental fractal (random Cantor set), for which the Hausdorff dimension is the most irrational number $\phi=(\sqrt{5}-1) / 2$, is used. Our models are based on the theory of fractional calculus [4] and the concept of a fractal. Also, there is the task of describing the super-nonradiative states (SNS) of various fields: gravitational, relic photons, the Higgs field, the neutrino field, the physical vacuum. Dynamic models make it possible to describe not only the superradiance, but also SNS. In [1] for the radiation intensity $J(t)$ was written

$$
J(t)=J_{0}\left(a_{0}+a_{m}\right)\left[\left(a_{0}-a_{m}\right)+1\right] .
$$

Here $J_{0}$ is the initial radiation intensity; in general case $a_{0}(t), a_{m}(t)$ depend on time $t$ and other parameters. The SNS we call the states with $J(t)=0$. These states can be realized in the process of development of various transient effects (induction, avalanche, echo, selfinduced transparency). These states can be realized in two models.

Model $\mathrm{A}_{0}$. Within this model, we assume $a_{0}=-a_{m}$, where

$$
a_{m}=\left(z_{A 2}^{\prime}\right)^{1 / 2} ; \quad a_{0}^{2}=a_{m}^{2}+z_{\mu}^{\prime}\left(z_{\mu}^{\prime}+2\right) / 4 ; \quad a_{m}^{2}=z_{A 2}^{\prime} ; \quad N_{r a}=z_{A 2}^{\prime}+z_{\mu}^{\prime},
$$

Here $z_{2 A}^{\prime}, z_{\mu}^{\prime}, N_{r a}$ are the usual redshift, cosmological redshift, total number of relic photons, respectively. From (2) it follows that two variants are possible: variant B1, when $z_{\mu}^{\prime}=0$, then $N_{r a}=z_{A 2}^{\prime}$; variant B2, when $z_{\mu}^{\prime}=-2$, then $N_{r a}=z_{A 2}^{\prime}-2$.

In model $\mathrm{A}_{0}$ the energy of the gravitational field $E_{0 A}=4.3110733 \mathrm{eV}$ is close to the

\footnotetext{
${ }^{*}$ Corresponding author: vsabramov@gmail.com
} 
boundary energy $4.3 \mathrm{eV}$, which is observed during neutrinoless double $\beta$-decay in experiments with the ${ }^{136} \mathrm{Xe}$ isotope [5]. This makes it possible to interpret these energies as the rest energy of Majorana neutrino. Direct experiments (DAMA/LIBRA, CoGeNT, CRESST-II collaborations) [3] by observing the spectrum and the angular distribution $\gamma$ radiation, the modulation spectrum from the galactic center show the following features: at the background of stochastic behavior the main local maximum near $2.4 \mathrm{keV}$ and two local main minimums near $1.9 \mathrm{keV}, 2.7 \mathrm{keV}$ are observed. In our model, the calculated energy $\varepsilon_{d m 4}=1.9053559 \mathrm{keV}, \varepsilon_{d m 4}^{\prime}=2.6945803 \mathrm{keV}$, which correspond to the local minima of the optical energy branches for dark matter with rest energy $\varepsilon_{d m}=1.7872164 \mathrm{keV}$, $\varepsilon_{d m}^{\prime}=2.5275057 \mathrm{keV}$, practically coincide with the position of the indicated local minima in the experimental modulation spectrum. The energy value $\varepsilon_{d m 3}^{\prime}+\varepsilon_{d m 2}=2.4093661 \mathrm{keV}$ almost coincides with the position of the main local maximum.

Model $\mathrm{A}_{1}$. In this model, we assume $a_{0}=a_{m}-1$. This condition makes it possible to introduce distribution density functions (DDF) $a_{m}, a_{m}^{\prime}$ for particles of Fermi type and DDF $N_{z g}, N_{z g}^{\prime}, n_{z g}, n_{z g}^{\prime}$ for particles of Bose type

$$
\begin{gathered}
a_{m}+a_{m}^{\prime}=1 ; \quad 2 a_{m}=1-b_{m} ; 2 a_{m}^{\prime}=1+b_{m} ; \quad 4 b_{m}=z_{\mu}^{\prime}\left(z_{\mu}^{\prime}+2\right) ; \\
N_{z g}^{\prime}-N_{z g}=1 ; \quad N_{z g}^{\prime}=n_{g} /\left[4-z_{\mu}^{\prime}\left(z_{\mu}^{\prime}+2\right)\right] ; \quad n_{z g}^{\prime}-n_{z g}=1 ; \quad n_{z g}^{\prime}=n_{g} /\left[4+z_{\mu}^{\prime}\left(z_{\mu}^{\prime}+2\right)\right] .
\end{gathered}
$$

The parameter $b_{m}$ (which can be interpreted as the population difference for particles of Fermi type) confirms the presence of a super-state, which is associated with $z_{\mu}^{\prime}$. Expressions (3) are written when the condition $-1 \leq b_{m} \leq 1$ is obtained. The occupation numbers $N_{z g}^{\prime}, n_{z g}^{\prime}$ confirm the possibility of describing SNS (dark matter) using the gluon field $\left(n_{g}=8\right)$, renormalized by contributions due to $z_{\mu}^{\prime}$. At $\left|b_{m}\right| \geq 1$ makes it possible to determine a frequency $v_{z g}^{\prime}=n_{z g}^{\prime} v_{G 0}=335.00053 \mathrm{MHz}$ close to the frequency $330 \mathrm{MHz}$, at which dark matter dominates from observations of radiofilaments [3]. Here $n_{z g}^{\prime}=0.114317037$ is DDF for particles of Fermi type $n_{z g}^{\prime}+n_{z g} \models 1, v_{G 0}=2.9304515 \mathrm{GHz}$ is characteristic frequency. It is shown (model $\mathrm{A}_{0}$ ), that information on the presence of SNS, dark matter, dark relic photons can be extracted from experimental spectra of $\gamma$-radiation by the presence of local minima on the background of the stochastic behavior of signals. It is possible (model $A_{1}$ ) to describe the processes of mutual transformations of particles of Bose and Fermi types. This can be used in neutrino physics, neuromedicine.

\section{References}

1. V.S. Abramov, Bull. of the Russian Academy of Sciences. Phys. 83, 3 (2019).

2. M.S. El Naschie, J. of Modern Physics. 7 (2016).

3. D. Hooper, Dark Universe. 1 (2012).

4. V.S. Abramov, Applied Non-Linear Dynamical Systems (Springer. 93, 2014).

5. R.M. Barnett et al., Phys. Rev. D54, 1 (1996). 